\title{
Treatment Policy for Grade IV and V Patients
}

\author{
Dae Hee Han, M.D., and Chang Wan Oh, M.D.
}

Department of Neurosurgery, Seoul National University College of Medicine, Seoul, South Korea

\begin{abstract}
To settle the controversy over optimum management strategy for patients with poor-grade (Hunt and Hess grade IV and V) aneurysmal subarachnoid hemorrhage (SAH), the medical records of 50 patients admitted with poor Hunt and Hess grade have been examined retrospectively with literature review. Initial findings such as better neurological condition (Hunt and Hess grade IV) and presence of intracerebral hemorrhage were indicators of good prognosis. Patients with intraventricular hemorrhage and uncontrollably increased intracranial pressure $\left(>40 \mathrm{~cm} \mathrm{H} \mathrm{H}_{2} \mathrm{O}\right.$ after extraventricular drainage) resulted in severe morbidity or mortality. Regarding the timing of surgery, early intervention led to a better outcome with less morbidity because of the marked decrease of the severity of vasospasm and the incidence of rebleeding. The frequency of intraoperative premature rupture and the overall mortality were not significantly influenced by the timing of surgery.

It is concluded that, for patients in poor Hunt and Hess grade without a definite evidence of irreversibility such as brainstem failure signs or uncontrollably increased intracranial pressure, early surgery followed by aggressive treatment to prevent cerebral vasospasm is the treatment of choice.
\end{abstract}

(Received \& accepted September 12, 1994)

Key words : aneurysm, subarachnoid hemorrhage, grade IV and V patients, early aneurysm surgery

Jpn J Neurosurg (Tokyo) 4 : 219-224, 1995

\section{Introduction}

Usually, we decide the management plan for patients with aneurysmal subarachnoid hemorrhage (SAH) according to their clinical condition. Hunt and Hess ${ }^{15)}$ categorized patients with SAH into grades according to their clinical status to standardize the treatment planning and prognostication, and presently this grading system is widely applied to diagnosis and treatment of aneurysmal SAH patients. With the advances in microneurosurgical techniques and improved preoperative and postoperative medical care, early surgery in low risk patients (Hunt and Hess grade I to III) is considered as a routine proce-

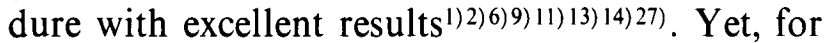
those patients with Hunt and Hess grade IV and
$\mathrm{V}$, there are still many controversies over the principle of management. The outlook for these patients has been dismal with the management mortality of $50 \%$ to $79 \%^{3 / 4)(5) 17) 23)}$ and they have been excluded from active treatment categories, including early surgery ${ }^{7724}$. Nevertheless recent advances in the microsurgical technique and postoperative management prompted us to reconsider the management principle for these poor grade patients.

\section{Determination of reversibility}

In the management of grade IV and V patients, the first and most important step is to determine if the harmful effect of SAH is reversible or not. Of course, only those patients with expected rever-

Address reprint requests to : Dae Hee Han, M.D., Department of Neurosurgery, Seoul National University College of Medicine, 28 Yongon-Dong, Chongno-Gu, Seoul 110-744, South Korea 
Table 1 Hunt and Hess grade and outcome

\begin{tabular}{crrrr}
\hline Hunt-Hess & \multicolumn{3}{c}{ Outcome scale } & \multirow{2}{*}{ Total } \\
\cline { 2 - 4 } grade & G. R. & Morb. & \multicolumn{1}{c}{ Mort. } & \\
\hline IV & $12(32)$ & $9(23)$ & $17(45)$ & $38(100)$ \\
V & $1(8)$ & $2(17)$ & $9(75)$ & $12(100)$ \\
\hline Total & $13(26)$ & $11(22)$ & $26(52)$ & $50(100)$ \\
\hline G. R. : good result & Morb. : morbidity \\
Mort. : mortality & $($ ) $: \%$
\end{tabular}

sibility can possibly benefit from active treatment, consisting of early surgery for aneurysmal neck clipping and aggressive postoperative management to prevent cerebral vasospasm.

Many authors agreed to that poor grade patients with significant intracerebral hematoma (ICH) have chances of good recovery by aggres-

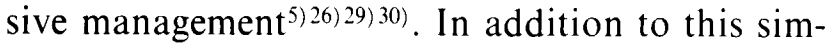
ple rule, a systematic approach to determine the reversibility was suggested by Dr. Bailes and Spetzler ${ }^{4}$. They managed patients with poor grade SAH based on radiographic and intracranial pressure (ICP) criteria rather than neurologic conditions. They utilized brain CT scanning to evaluate the brain status such as signs of irreversible brain destruction; massive cerebral infarction with midline shift, dominant basal ganglia or brain stem hematoma. Poor of absent intracranial vascular filling on CT or cerebral angiography and increased intracranial pressure (ICP) above $30 \mathrm{~cm} \mathrm{H}_{2} \mathrm{O}$ without an intracranial clot or above $50 \mathrm{~cm} \mathrm{H} \mathrm{H}_{2} \mathrm{O}$ in the presence of a clot after extraventricular drainage were also regraded as a predictor of irreversibility. In their report, only those patients without an evidence of irreversible damage underwent craniotomy for aneurysmal neck clipping and hematoma removal followed by aggressive postoperative triple H's (hypertensive, hypervolemic and hemodilutional) therapy, and $54.3 \%$ of them resulted in good recovery. Another unpublished series also utilized CT scanning and ICP to determine reversibility ${ }^{22)}$. In that series, destruction of vital structure and ICP above $20 \mathrm{~cm} \mathrm{H}_{2} \mathrm{O}$ were regarded as indicators of irreversibility.

Other possible means to determine reversibility includes measurement of cerebral blood flow and somatosensory and / or auditory evoked potentials ${ }^{10)(2) 31)}$, whether or not they are practical in the management of poor-grade patients.

Our data also showed that the presence of ICH and controllable ICP are good indicators of reversibility. Especially, all patients with ICP above 40 $\mathrm{cm} \mathrm{H}_{2} \mathrm{O}$ died regardless of any treatment modalities.

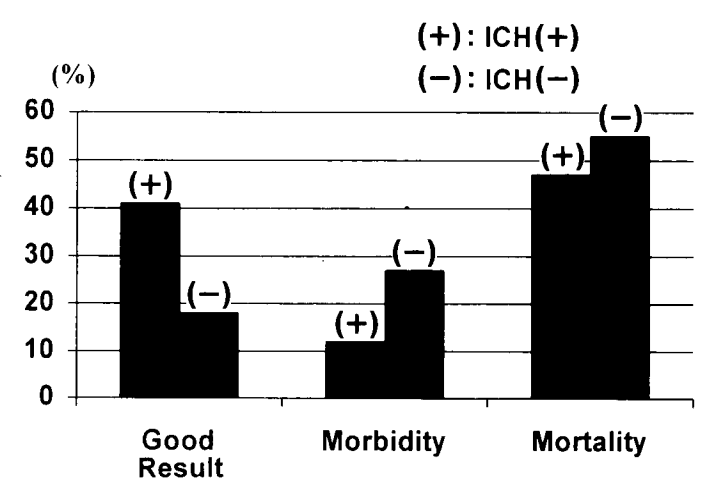

Fig. 1 Intracerebral hemetoma and outcome Patients with intracerebral hematoma demonstrate better outcome.

$\mathrm{ICH}$ : intracerebral hemorrhage

\section{Prognostic factors}

There are several clinical factors that are helpful in predicting the outcome of treatment in

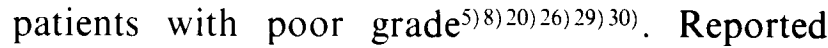
poor prognostic factors include poor neurologic condition (especially consciousness level), old age, high initial systolic blood pressure, large aneurysm size, deep location of aneurysm (anterior communicating and posterior circulation aneurysms), repeated bleeding, intraventricular hemorrhage (IVH) and severe SAH (especially in perimesencephalic cistern). Meanwhile, ICH or acute hydrocephalus, that causes poor neurological condition by mass effect and are amenable to surgical treatment, have been considered as good prognostic factors.

To confirm the prognostic effects of these factors, we performed retrospective analysis of 50 poor-grade patients, consisting of 38 grade IV and 12 grade $\mathrm{V}$ patients. From the data presented here (Table 1) and in most reports, it is obvious that a distinct difference exists in regards to the overall morbidity and mortality between patients admit-

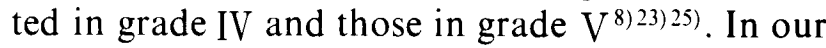
series of grade $V$ patients, the outcome was disappointing with either severe morbidity or mortality in $92 \%$, which is in good agreement with other reports.

On admission, 17 case of our series showed $\mathrm{ICH}$, and they resulted in better outcome with higher good results and less morbidity and mortality than those without ICH (Fig. 1). Other authors also agreed to that the presence of $\mathrm{ICH}$ is related to chances of good recovery ${ }^{526) 29)}$ 30). Yet, some other report failed to demonstrate such an positive association between ICH and good outcome $^{8)}$. Probably, this reflects that the effect of 


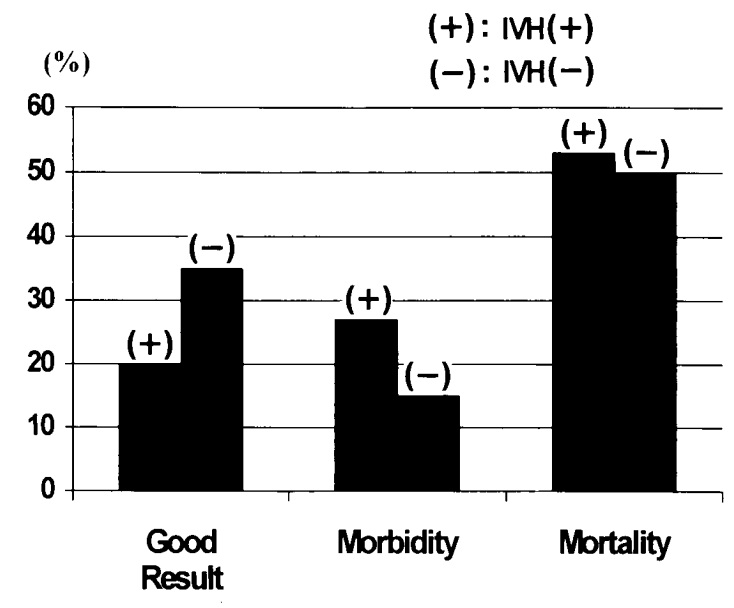

Fig. 2 Intraventricular hemorrhage and outcome

Intraventricular hemorrhage was a predictor of poor prognosis.

IVH : intraventricular hemorrhage

Table 2 Location of aneurysm and outcome

\begin{tabular}{lcccc}
\hline \multicolumn{1}{c}{ Result } & ACA $^{*}$ & ICA & MCA & Post. Circ. \\
\hline G. R. & $2(18)$ & $6(35)$ & $4(44)^{* *}$ & $1(33)$ \\
Morb. & $4(36)$ & $5(30)$ & $1(11)$ & $1(33)$ \\
Mort. & $5(45)$ & $6(35)$ & $4(44)$ & $1(33)$ \\
\hline Total & $11(100)$ & $17(100)$ & $9(100)$ & $3(100)$ \\
\hline${ }^{*} 9$ cases $(81 \%)$ & with IVH & ${ }^{* *}$ All with sylvian \\
hematoma ACA : anterior communicating \\
artery ICA : internal carotid artery \\
MCA : middle cerebral artery Post. Circ. \\
posterior circulation $\quad(\quad): \%$
\end{tabular}

ICH on outcome may change depending on its location and mode of management, which may vary from one series to the other.

Intraventricular hemorrhage (IVH) was present in $60 \%$ of the patients. It served as a bad prognostic factor (Fig. 2), leading to higher morbidity and less good results, in accordance with other reports ${ }^{821)}$. Acute hydrocephalus, which was shown in 29 cases (58\%) on initial CT scan, demonstrated no definite correlation with outcome in our series.

Regarding the location of ruptured aneurysm, middle cerebral artery aneurysms showed higher good result and mortality compared with those in other locations (Table 2). This means that poorgrade patients with ruptured middle cerebral artery aneurysm tend to have large sylvian hematoma and/or IVH which may be fatal ${ }^{18)}$, but it can also indicate higher chances of good recovery with less morbidity if the hematoma is evacuated promptly ${ }^{28)}$.

The effect of initial ICP on outcome was definite in our series. The initial ICP after extraventricular drainage was above $40 \mathrm{~cm} \mathrm{H}_{2} \mathrm{O}$ in
Table 3 Management mode and outcome

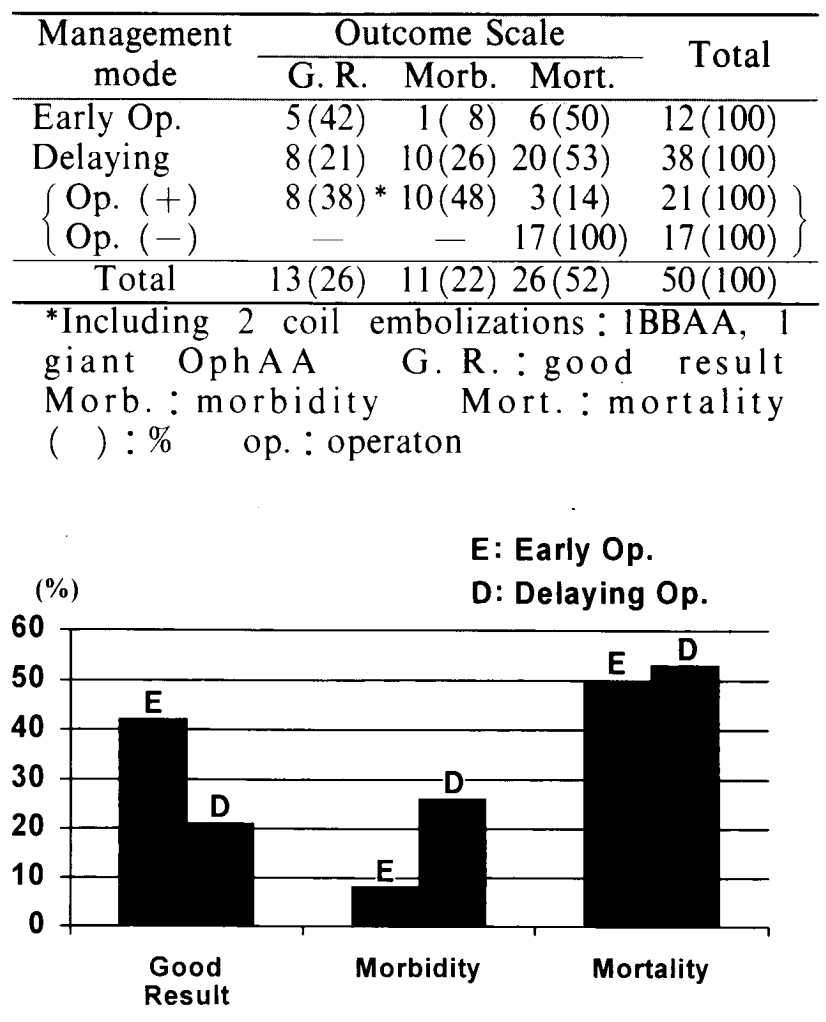

Fig. 3 Management mode and outcome

Early surgery group shows higher good result with less morbidity.

5 patients. One patient was managed by early surgery, another one with delayed surgery, and the other 3 underwent no operation. All of these 5 patients died regardless of management modality.

Other factors such as patients' age, sex, usage of nimodipine and initial blood pressure showed no definite influence on outcome.

\section{Timing of surgery and outcome}

Patients with poor-grade SAH show higher rebleeding rate in acute stage ${ }^{16)}$ and higher risk of clinical vasospasm ${ }^{19}$, which together with severe initial brain damage lead to poor outcome. For these patient group with dismal outlook, there still remains controversy over the optimum timing of surgery4)7) 11) 24)25).

It is well known that early surgery for patients with aneurysmal SAH has several advantages over delayed one. It can immediately eliminate some factors contributing to poor neurologic condition (such as ICH and hydrocephalus) and prevent rebleeding, allowing the removal of subarachnoid hemorrhage (so-called "cisternal toilet") and aggressive postoperative management to prevent cerebral vasospasm. Meanwhile, early 
Table 4 Causes of mortality

\begin{tabular}{|c|c|c|c|c|c|}
\hline $\begin{array}{c}\text { Monagement } \\
\text { mode }\end{array}$ & $\begin{array}{c}\text { Initial } \\
\text { damage }\end{array}$ & $\begin{array}{l}\text { Symptomatic } \\
\text { vasospasm }\end{array}$ & Rebleeding & Others & Total \\
\hline Early operation & $4(67)$ & $1(17)$ & - & $1(17)$ & $6(100)$ \\
\hline Delaying & $7(35)$ & $5(25)$ & $7(35)$ & $1(5)$ & $20(100)$ \\
\hline operation $(t)$ & - & $2(66)$ & $1(33)$ & - & $3(100)$ \\
\hline operation $(-)$ & $7(41)$ & $3(18)$ & $6(35)$ & $1(6)$ & $17(100)$ \\
\hline Total & $11(42)$ & $6(23)$ & $7(27)$ & $2(8)$ & $26(100)$ \\
\hline
\end{tabular}

Table 5 Causes of morbidity

\begin{tabular}{|c|c|c|c|c|c|}
\hline $\begin{array}{l}\text { Management } \\
\text { mode }\end{array}$ & $\begin{array}{l}\text { Initial } \\
\text { damage }\end{array}$ & $\begin{array}{l}\text { Symptomatic } \\
\text { vasospasm }\end{array}$ & Rebleeding & Others & Total \\
\hline Early operation & - & $1(100)$ & - & - & $1(100)$ \\
\hline Delaying & $1(10)$ & $7(70)$ & $1(10)$ & $1(10)$ & $10(100)$ \\
\hline$\{$ operation $(+)$ & $1(10)$ & $7(70)$ & $1(10)$ & $1(10)$ & $10(100)$ \\
\hline operation $(-)$ & - & - & - & - & - \\
\hline Total & $1(9)$ & $8(73)$ & $1(9)$ & $1(9)$ & $11(100)$ \\
\hline
\end{tabular}

Table 6 Incidence of vasospasm

\begin{tabular}{|c|c|c|}
\hline $\begin{array}{l}\text { Management } \\
\text { mode }\end{array}$ & $\begin{array}{c}\text { Symptomatic } \\
\text { vasospasm }\end{array}$ & $\begin{array}{c}\text { Severe* } \\
\text { vasospasm }\end{array}$ \\
\hline Early Operation & $6(50)$ & $3(25)$ \\
\hline Delaying & $21(55)$ & $14(37)$ \\
\hline $\int$ operation $(+)$ & $16(76)$ & $11(52)$ \\
\hline operation $(-)$ & $5(29)$ & $3(18)$ \\
\hline Total & $26(52)$ & $17(34)$ \\
\hline
\end{tabular}

*Symptomatic vasospasm leading to morbidity or mortality ( ) :\%

surgery also has some drawbacks, especially in the cases of poor-grade SAH. It may cause additional damage to the brain by surgical manipulation and increase the incidence of premature rupture and cerebral vasospasm.

We analyzed 50 consecutive patients with Hunt and Hess grade IV-V to evaluate the effect of early surgery (operation within 72 hours of SAH) on outcome. The patients were divided into two groups: those with early surgery and the others without early surgery. The latter group was called "delaying operation group" in our study. Management morbidity and mortality, the incidence of vasospasm, rebleeding and intra-operative aneurysm rupture were compared between these two groups. The outcomes of treatment were classified into three categories according to the Glasgow Outcome Scale on discharge, that is, good result (good recovery or moderately disabled), morbidity (severely disabled or vegetative) and mortality.

Twelve patients (24\%) underwent early surgery and, 19 patients $(38 \%)$ were treated by delayed

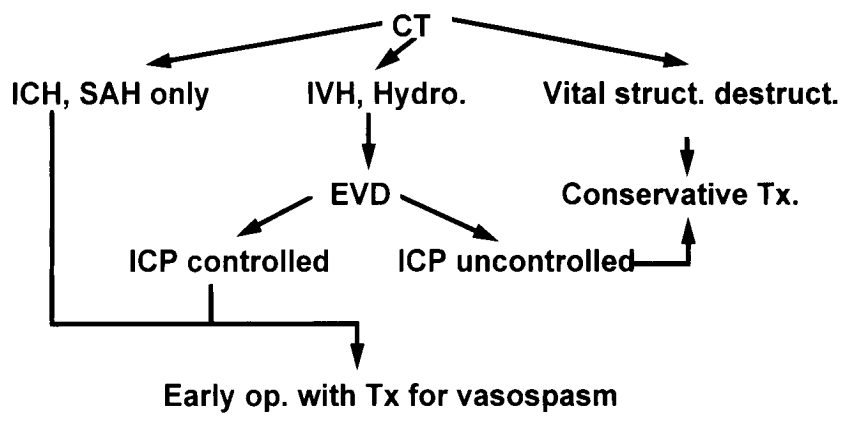

Fig. 4 Recommended treatment protocol for patients with poor-grade SAH

surgery, while the remaining 19 (38\%) received no operation. After aneurysmal neck clipping, all patients were managed by active triple H's therapy. The management outcome demonstrated higher good result and lower morbidity in early surgery group compared with those managed by delaying operation (Table 3, Fig. 3). Both groups resulted in nearly equal mortality rate of about $50 \%$. All patients managed without surgery died.

According to the mode of management, the causes of mortality and morbidity were analyzed (Table 4, 5). In early operation group, the major cause of death was initial brain damage by aneurysmal rupture. Meanwhile, in delaying operation group, nearly half of the patients died of rebleeding or vasospasm. Regarding morbidity, $70 \%$ of morbidity came from vasospasm in delaying operation group. These results indicated that vasospasm or rebleeding are major causes of mortality and morbidity in delaying operation group.

As to vasospasm, the overall incidence of symptomatic vasospasm was not significantly different between those two management groups (Table 6). 
Nevertheless, the incidence of severe vasospasm, leading to mortality or morbidity, was higher in the delaying operation group. In addition to severe vasospasm, 11 patients $(29 \%)$ of the delaying operation group suffered from rebleeding, and 10 of them died or resulted in serious morbidity.

In delaying operation group, the Hunt and Hess grade improved during supportive management in only 4 patients (11\%). The incidence of intraoperative premature rupture was about $10 \%$, and it was not significantly different between the two management groups.

In summary, our analysis revealed that the management mortality was similar in both management groups, but the morbidity was higher in the delaying operation group mainly due to vasospasm. With regard to symptomatic vasospasm, the overall incidence was similar in both groups, but it was more severe in the delaying operation group. The overall management mortality was $52 \%$ and the morbidity was $8 \%$ in early surgery group and $26 \%$ in delaying operation group.

\section{Conclusion and treatment protocol}

With the above analysis, we could conclude that early surgery for poor-grade SAH patients improves outcome with less morbidity by decreasing the severity of vasospasm and preventing rebleeding. The incidence of symptomatic vasospam, intra-operative premature rupture and mortality were not significantly different between early surgery group and those managed by delaying operation.

Therefore, for patients with poor Hunt and Hess grade, early surgery and aggressive treatment to prevent cerebral vasospasm may be the choice of treatment, except those cases with definitely unmanageable factors, such as the destruction of vital structure or uncontrollably increased intracranial pressure.

Fig. 4 shows recommended protocol of treatment for poor-grade SAH patients based on the analysis of our series.

\section{References}

1) Adams HP : Early management of the patients with recent aneurysmal subarachnoid hemorrhage. Stroke 17: 1068-1070, 1986.

2) Adams HP, Kassel NF, Kongable GA, Torner JC: Intracranial operation within seven days of aneurysmal subarachnoid hemorrhage. Results in 150 patients. Arch Neurol $45: 1065-1069$, 1988.
3) Adams HP Jr, Kassel NF, Torner JC, Nibbelink DW, Sahs AL : Early management of aneurysmal subarachnoid hemorrhage. A report of the cooperative aneurysm study. $J$ Neurosurg 54:141-145, 1981.

4) Bailes JE, Spetzler RF, Hadley MN, Baldwin $\mathrm{HZ}$ : Management morbidity and mortality of poor grade aneurysm patients. $J$ Neurosurg 72: 559-566, 1990.

5) Brandt I, Sonesson B, Ljunggren B, Säveland $\mathrm{H}$ : Ruptured middle cerebral artery aneurysm with intracerebral hemorrhage in younger patients appearing moribund : emergency operation? Neurosurgery 20:925-929, 1987.

6) Chyatte D, Fode P, Sundt TM Jr : Early versus late intracranial aneurysm surgery in subarachnoid hemorrhage. $J$ Neurosurg $69: 326-331$, 1988.

7) Deruty R, Mottolese C, Guyotat IP, Soustiel $\mathrm{JF}$ : Management of the ruptured intracranial aneurysm-early surgery, late surgery, or modulated surgery? Acta Neurochir (Wien) 113: 1-10, 1991.

8) Disney L, Weir B, Grace $M$ and Canadian Nimodipine Study Group : Factors influencing the outcome of aneurysm rupture in poor grade patients: A prospective series. Neurosurgery $23: 1-8,1988$.

9) Disney L, Weir B, Pretru K : Effect on management mortality of a deliberate policy of early operation on supratentorial aneurysms. Neurosurgery $20: 695-701,1987$.

10) Fazl M, Houlden DA, Weaver K : Correlation between cerebral blood flow, somatosensory evoked potentials, CT scan grade and neurological grade in patients with subarachnoid hemorrhage. Can J Neurol Sci 18:453-457, 1991.

11) Hernesniemi J, Vapalahti $M$, Niskanen $M$, Tapaninaho A, Kari A, Luukkonen M, Puranen M, Saari T, Rajpar M : One-year outcome in early aneurysm surgery. A 14 years experience. Acta Neurochir (Wien) 122:1-10, 1993.

12) Hojer C, Haupt WF : The prognostic value of AEP and SEP values in subarachnoid hemorrhage. An analysis of 64 patients. Neurochirrurgia (Stuttg) 36 (4) : 110-116, 1993.

13) Hori S, Suzuki J : Early intracranial operations for ruptured aneurysms. Acta Neurochir (Wien) 46:93-104, 1979.

14) Hugenholtz $\mathrm{H}$, Elge $\mathrm{R}$ : Considerations in early surgery on good-risk patients with ruptured intracranial aneurysms. $J$ Neurosurg $29: 14$ $-20,1982$.

15) Hunt WE, Hess RM : Surgical risk as related to time of intervention in the repair of intracranial aneurysms. J Neurosurg 28: 14-20, 1968.

16) Inagawa $T$, Kamiya K, Ogasawara $H$, Yano $T$ : Rebleeding of ruptured intracranial aneurysms in the acute stage. Surg Neurol 28 (2) : 93-99, 1987. 
17) Inagawa $T$, Takahashi $M$, Hidenobu A, Ishikawa S, Yoshimoto $\mathrm{H}$ : Aneurysmal subarachnoid hemorrhage in Izumo city and Shimane Prefecture of Japan-Outcome. Stroke 19: 176-181, 1988.

18) Karhunen PJ, Servo A : Sudden fatal or nonoperable bleeding from ruptured intracranial aneurysm. Evaluation by post-mortem angiography with vulcanishing contrast medium. Int J Legal Med 106 (2) : 55-59, 1993.

19) Mendel RC, Carter LP: Evaluation and treatment of clinical vasospasm following subarachnoid hemorrhage. Contemp Neurosurg 16 (6) : 1-6, 1994.

20) Mizuno M, Yasui N, Suzuki A, Hadeishi H, Nakajima S, Sampei T, Ohtsuki H : Clinical course in poor grade patients with ruptured intracranial aneurysms and extensive subarachnoid clot. No Shinkei Geka 20:21-29, 1992.

21) Mohr G, Ferguson G, Khan M, Malloy D, Watts R, Benoit B, Weir B: Intraventricular hemorrhage from ruptured aneurysm. Retrospective analysis of 91 cases. $J$ Neurosurg $58: 482-487,1983$.

22) Ogilvy CS, Gress DR, Choi IS, Crowell RM : Mechanism of neurological impairment in poor - grade aneurysm patients and consideration of early aneurysm treatment. AANS Abstract, Poster\#1122, 1994.

23) Petruk KC, West M, Mohr G, Weir BKA, Benoit BG, Gentili F, Disney L, Khan MI, Grace M, Holness RO, Karwon MS, Ford RM, Cameron GS, Tucker WS, Purves GB, Miller JDR, Hunter KM, Richard MT, Durity FA, chan R, Clein LJ, Maroun FB, Godon A : Nimodipine treatment in poor grade aneurysm patients. Results of a multicenter double-blind placebo-controlled trial. $J$ Neurosurg 68 : 505-517, 1988.

24) Sano K, Asano T, Tamura A : Acute aneurysm surgery. Physiopathology and management. Wien, New York, Springer, 1987, pp.289.

25) Seifert V, Trost HA, Stolke D : Management morbidity and mortality in grade IV and $\mathrm{V}$ patients with aneurysmal subarachnoid hemorrhage. Acta Neurochir (Wien) 103:5-10, 1990.

26) Sundt TM Jr, Whisnant JP: Subarachnoid hemorrhage from intracranial aneurysms. Surgical management and natural history of disease. $N$ Engl J Med 229:116-122, 1978.

27) Suzuki J, Onuma T, Yoshimoto T : Results of early operation on cerebral aneurysm. Surg Neurol 11: 407-412, 1979.

28) Tapaninaho A, Hernesnieme J, Vapalahti M : Emergency treatment of cerebral aneurysms with large hematomas. Acta Neurochir (Wien) 91 (12) : 21-24, 1988.

29) Vermeullen $M$, van Gijn J, Hijdra A, Crevel $\mathrm{H}$ : Causes of acute deterioration in patients with a ruptured intracranial aneurysm. $J$ Neurosurg 60:935-939, 1984.

30) Wheelock B, Weir B, Watts R, Mohr G, Khan M, Hunter M, Fewer D, Fersuson G, Durity F, Cochrane D, Benoit B: Timing of surgery for intracranial hematomas due to aneurysm rupture. J Neurosurg 58:476-481, 1983.

31) Yamagata S, Kikuchi $H$, Ihara I, Nagata I, Morooka Y, Naruo Y, Koizumi T, Hashimoto $\mathrm{K}$, Minamikawa J, Miyamoto $\mathrm{S}$ : Cerebral blood flow in subarachnoid hemorrhage: analysis in patients with poor outcomes. No Shinkei Geka 15: 1189-1196, 1987. 